\title{
Trigeminal Schwannoma
}

National Cancer Institute

\section{Source}

National Cancer Institute. Trigeminal Schwannoma. NCI Thesaurus. Code C4655.

A benign schwannoma occurring in the trigeminal nerve. 